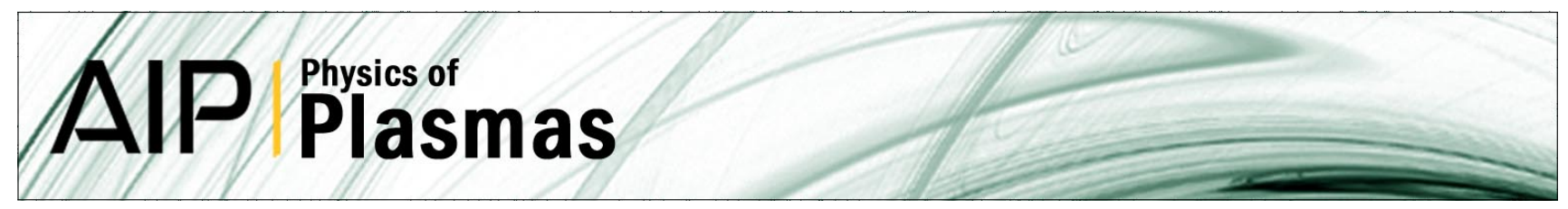

\title{
Isothermal, mass-limited rarefactions in planar and spherical geometry
}

R. Paul Drake

Citation: Phys. Plasmas 18, 104506 (2011); doi: 10.1063/1.3642612

View online: http://dx.doi.org/10.1063/1.3642612

View Table of Contents: http://pop.aip.org/resource/1/PHPAEN/v18/i10

Published by the AIP Publishing LLC.

\section{Additional information on Phys. Plasmas}

Journal Homepage: http://pop.aip.org/

Journal Information: http://pop.aip.org/about/about_the_journal

Top downloads: http://pop.aip.org/features/most_downloaded

Information for Authors: http://pop.aip.org/authors

\section{ADVERTISEMENT}

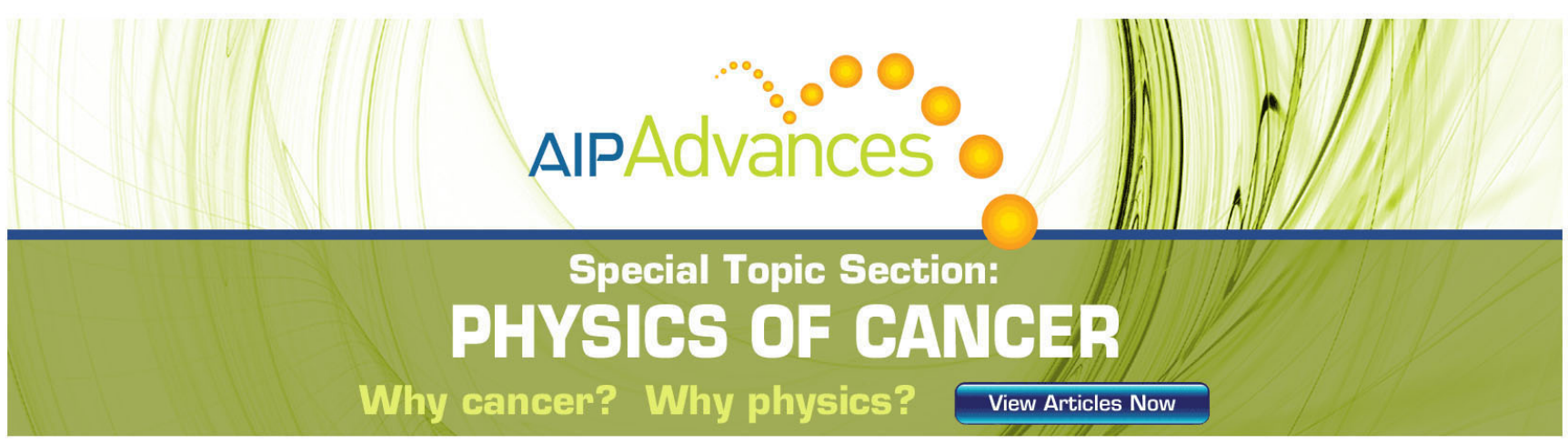




\title{
Isothermal, mass-limited rarefactions in planar and spherical geometry
}

\author{
R. Paul Drake ${ }^{a)}$ \\ University of Michigan, Ann Arbor, Michigan 48105, USA
}

(Received 13 April 2011; accepted 28 August 2011; published online 14 October 2011)

\begin{abstract}
Solutions are developed for isothermal, mass-limited rarefactions in which the temperature is held constant by continued heating. The case of spatially constant temperature and fixed total energy is also revisited. These solutions are useful in the context of experiments in which plasmas are continuously heated for long periods (up to tens of ns) by present-day lasers or hohlraums.
\end{abstract}

(C) 2011 American Institute of Physics. [doi:10.1063/1.3642612]

The expansion of plasmas is of fundamental importance in a wide range of circumstances, for example, including laser-heated systems ${ }^{1}$ and supernova explosions. ${ }^{2}$ This lends value to simple models of such expansions, from the very basic, self-similar, adiabatic and isothermal rarefactions of semi-infinite slabs often discussed in books ${ }^{3,4}$ to other simple models discussed in the literature. In particular, see Qi and Krishnan $^{5}$ and references therein. Much of this work ${ }^{6,7}$ was motivated by the exploding laser targets used for $\mathrm{x}$-ray lasers and for laser-plasma-interaction studies, ${ }^{8}$ and there has some $^{9}$ but been relatively little work since 1990 on singlefluid expansions. There is more recent work on two-fluid expansions, ${ }^{10,11}$ in which some electrons eventually pull ahead of the ions, motivated by particle acceleration from surfaces driven by high-irradiance lasers. Here, however, we are concerned with the behavior away from the ion front and thus with single-fluid models.

An aspect of the prior work is the absence of models for the expansion of a constant-temperature plasma of finite mass, save for the approximate result given by London and Rosen. ${ }^{6}$ Yet this case is of some relevance, both for a period of time in exploding-foil targets and for the expansion of plasma of finite mass whose temperature is sustained in some way, such as by a hohlraum or by a radiative shock. Electron heat conduction is quite rapid in densities below solid density, so that constant-temperature models are often sensible approximations. The present brief communication is intended to address this finite mass, constant-temperature regime. We refer below to the paper ${ }^{6}$ by London and Rosen as LR, to the paper ${ }^{5}$ by Qi and Krishnan as QK, and to the paper $^{7}$ by Hunter and London as HL.

Before beginning a calculation, it is worthwhile to recall some of the relevant physical context. The single fluid treatment is justified as follows. In the expanding plasma, the electron fluid motion is continuously in steady state relative to that of the ion fluid, establishing an electric field $E$ of magnitude given by $e n_{e} E=-\nabla p_{e}$, where the electron charge, number density, and pressure are $e, n_{e}$, and $p_{e}$, respectively. This electric field in turn accelerates the ions with the effect that the net source in the equation for the momentum density is $-\nabla p$, with $p$ being the total pressure. The

\footnotetext{
${ }^{a)}$ Electronic mail: rpdrake@umich.edu.
}

importance of the cases with spatially constant temperature considered here is that these are realistic because for typical conditions electron heat conduction is fast. One can find the timescale for electron heat conduction from

$$
\left.\rho C_{v} \frac{\partial T_{e}}{\partial t}\right|_{h c}=-\nabla \cdot \kappa \nabla T_{e},
$$

where the mass density, electron temperature, specific heat at constant volume, and heat conduction coefficient are $\rho$, $T_{e}, C_{v}$, and $\kappa$, respectively. For the fully ionized plasmas typically of interest here $C_{v}=(1+Z) k_{B} /\left(A m_{p}\right)$, where the average charge, average atomic number, proton mass, and Boltzmann constant are $Z, A, m_{p}$, and $k_{B}$, respectively, and

$$
\kappa=\frac{128}{3 \pi} \frac{n_{e} k_{B} T_{e}}{m_{e} \nu_{e i}} k_{B},
$$

in which the electron mass is $m_{e}$ and the electron-ion collision rate is $\nu_{e i}=Z \omega_{p e}^{4} \ln \Lambda /\left(6 \sqrt{2} \pi^{3 / 2} n_{e}\left(k_{B} T_{e} / m_{e}\right)^{3 / 2}\right)$, which in $\mathrm{s}^{-1}$ is $3 \times 10^{-6} \ln \Lambda n_{e} Z T_{e V}^{-3 / 2}$ for $n_{e}$ in $\mathrm{cm}^{-3}$ and $T_{e V}$ in $\mathrm{eV}$. (Note $k_{B}=1.6 \times 10^{-12} \mathrm{ergs} / \mathrm{eV}, \omega_{p e}$ is the electron plasma frequency, and $\ln \Lambda$ is the Coulomb logarithm.) From this one can find the ratio of the temperature equilibration timescale, $t_{\text {equil }}$, to the expansion timescale, $t_{\text {exp }}$. The equilibration timescale is defined in typical fashion from Eq. (1) as

$$
t_{\text {equil }}=\frac{\rho C_{v} L^{2}}{\kappa}
$$

while $t_{\text {exp }}$, the experiment duration, is the period of time during which the expansion has continued. This typically would be the time interval in an experiment from the initial deposition of energy to the time of observation. Here $L$ is the spatial scale of the expanding system at any $t_{\text {exp }}$, for which a basic estimate would be $t_{\text {exp }}$ times the sound speed. The ratio $t_{\text {equil }} / t_{\text {exp }}$, shown in Fig. 1, is proportional to $t_{\text {exp }}$, rather than to $1 / t_{\text {exp }}$, because of the factor $L^{2}$ in $t_{\text {equil }}$. Typical laser blowoff plasmas are below $10^{21} \mathrm{~cm}^{-3}$ in electron density. One can see that heat conduction will be rapid for most cases of interest.

We commence the derivation with the usual equations for the conservation of mass and momentum, being 


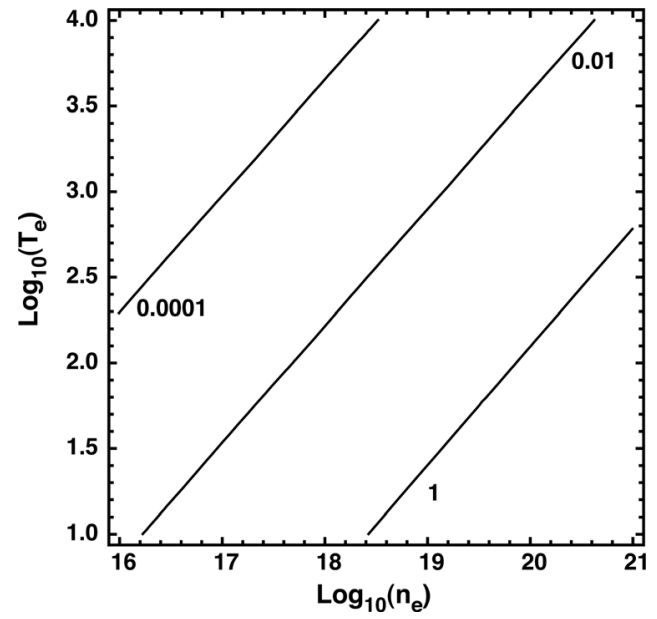

FIG. 1. This is the ratio of the electron temperature equilibration time to the experiment duration $t_{\text {exp }}$, evaluated for $t_{\text {exp }}=10 \mathrm{~ns}$ and for a fully ionized $\mathrm{Be}$ plasma. When this quantity is small, the plasma will remain isothermal.

$$
\begin{aligned}
& \frac{\partial \rho}{\partial t}+\nabla \cdot(\rho \mathbf{u})=0 \quad \text { and } \\
& \frac{\partial \mathbf{u}}{\partial t}+\mathbf{u} \cdot \nabla \mathbf{u}+\frac{1}{\rho} \frac{\partial p}{\partial r}=0,
\end{aligned}
$$

respectively, where $\rho$ is mass density, $\mathbf{u}$ is velocity, and $p$ is pressure. We seek here symmetric planar or spherical expansions, so $\mathbf{u}$ is radial and these equations become

$$
\begin{gathered}
\frac{\partial \rho}{\partial t}+u \frac{\partial \rho}{\partial r}+\rho\left(\frac{\partial u}{\partial r}+\frac{s u}{r}\right) \text { and } \\
\frac{\partial u}{\partial t}+u \frac{\partial u}{\partial r}+\frac{1}{\rho} \frac{\partial p}{\partial r}=0,
\end{gathered}
$$

where $s$ is zero for planar expansions and 2 for spherical ones.

For the assumed free, homogeneous expansion $u \propto r$ and we use the ultimately convenient form that

$$
u=\frac{1}{L} \frac{d L}{d t} r
$$

where the scale length $L$ is a function of $t$ only. We also use an ideal-gas equation of state with gas constant $R$ and temperature $T$ and assume $R$ and $T$ to be constant in space as is justified above. Then Eq. (7) becomes

$$
\frac{r L^{\prime \prime}}{L}+\frac{R T}{\rho} \frac{\partial \rho}{\partial r}=0
$$

where the prime indicates a derivative in time. This equation has spatial solution

$$
\rho=\hat{\rho} \exp \left[-\frac{r^{2} L^{\prime \prime}}{2 R T L}\right]
$$

where $\hat{\rho}$ is the time-dependent density at $r=0$. This maximum density is related to other quantities by conservation of total mass. For the planar case with areal mass density $\sigma$ one finds

$$
\hat{\rho}_{p l}=\sigma \sqrt{\frac{2}{\pi}} \sqrt{\frac{L^{\prime \prime}}{L R T}},
$$

while for the spherical case with total mass $M$ one finds

$$
\hat{\rho}_{s p h}=\frac{M}{(2 \pi)^{3 / 2}}\left(\frac{L^{\prime \prime}}{L R T}\right)^{3 / 2} .
$$

We find the implications of the mass equation by substituting Eqs. (8) and (11) or (12) and into Eq. (6) with the appropriate value for $s$. In both cases, one finds that the solution is $R T=L L^{\prime \prime}$. As a result the profiles become

$$
\begin{aligned}
& \hat{\rho}_{p l}=\sqrt{\frac{2}{\pi}} \frac{\sigma}{L} \exp \left[-\frac{r^{2}}{2 L^{2}}\right] \text { and } \\
& \hat{\rho}_{s p h}=\frac{M}{(2 \pi)^{3 / 2}} \frac{1}{L^{3}} \exp \left[-\frac{r^{2}}{2 L^{2}}\right] .
\end{aligned}
$$

These density profiles are well known but the unmet challenge is posed by the seemingly simple equation $R T=L L^{\prime \prime}$. We consider the case when $R T$ is held constant for all time by some heat source. This turns out to have a solution, which can be evaluated numerically. The solution to

$$
\frac{\partial}{\partial t}\left[L \frac{\partial^{2} L}{\partial t^{2}}\right]=0
$$

can be written

$$
L=\frac{L_{o}}{1.88} \exp \left[-\left(\operatorname{Erf}^{-1}\left[i \frac{2}{\sqrt{\pi}} \frac{t}{t_{o}}\right]\right)^{2}\right],
$$

where as we will see the initial state has $L=L_{o}$ at $t=t_{o}$ and the origin of the factor 1.88 is described below. To convert this to a more tractable form, one must further discuss the error function and its inverse. One has

$$
i z=\operatorname{Erf}^{-1}[\operatorname{Erf}(i z)]=\operatorname{Erf}^{-1}\left[i \frac{2}{\sqrt{\pi}} e^{z^{2}} D(z)\right]
$$

where

$$
\begin{gathered}
\operatorname{Erf}(x)=\frac{2}{\sqrt{\pi}} \int_{0}^{x} e^{-y^{2}} d y, \text { so } \\
\operatorname{Erf}(i z)=i \frac{2}{\sqrt{\pi}} \int_{0}^{z} e^{y^{2}} d y=i \frac{2}{\sqrt{\pi}} e^{z^{2}} D(z),
\end{gathered}
$$

where $D(z)$ is the Dawson function,

$$
D(z)=e^{-z^{2}} \int_{0}^{z} e^{y^{2}} d y .
$$

With this definition of $z$ we have

$$
L=\frac{L_{o} e^{z^{2}}}{1.88},
$$

where $z$ is a solution of

$$
\int_{0}^{z} e^{t^{2}} d t=\frac{t}{t_{o}}
$$




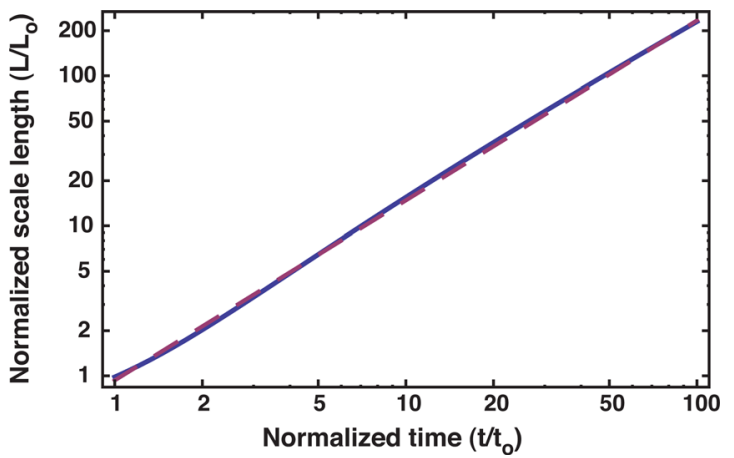

FIG. 2. (Color online) The ratio $L / L_{o}$ from Eq. (21) is shown against $t / t_{o}$, along with the fit $0.95\left(t / t_{o}\right)^{1.2}$.

The factor 1.88 is, to three-digit accuracy, the solution of Eq. (22) for $t=t_{o}$, assuring that $L=L_{o}$ for $t=t_{o}$. Figure $2 \mathrm{com}$ pares the solution for $L / L_{o}$ from Eqs. (21) and (22) with the fit

$$
L / L_{o}=0.95\left(t / t_{o}\right)^{1.2},
$$

which is accurate to better than $7 \%$ over the range $1 \leq t / t_{o} \leq 100$. Thus, the time dependent profiles are given by Eqs. (13) and (14) with $L$ from Eq. (23) or a numerical evaluation of Eq. (22), and one needs to know a value of $L$ at some initial time. One can also note that LR report a solution of Eq. (15), valid in the (very late time) limit that $\ln \left[t / t_{o}\right] \gg 1$, which can be written as

$$
L / L_{o}=\sqrt{2}\left(t / t_{o}\right) \sqrt{\ln \left[\sqrt{2}\left(t / t_{o}\right)\right]}
$$

To provide concrete examples, one may consider two possible experiments using large plasmas at the National Ignition Facility $^{12}$ (NIF). The goal of such experiments might be to produce collisionless and/or magnetized flows relevant to collisionless shocks or magnetization of blast waves, and indeed some experiments similar to these have been selected for NIF. In the design of such experiments, one is likely to focus on ion density rather than mass density, in order to evaluate relevant quantities such as ion skin depth or collision lengths, so Fig. 3 shows ion density. Using $1 \mathrm{MJ}$ of laser energy at a wavelength of $0.35 \mu \mathrm{m}$, NIF can irradiate a $5 \mathrm{~mm}$ diameter target with an irradiance of $10^{14} \mathrm{~W} / \mathrm{cm}^{2}$ for $50 \mathrm{~ns}$, or a larger area for a shorter time. For the case of a $\mathrm{C}$ target this will sustain an electron temperature near $2 \mathrm{keV}$, so that the sound speed is $330 \mu \mathrm{m} / \mathrm{ns}$. The mass ablation rate will be about 2.6 $\mu \mathrm{m} / \mathrm{ns}$. In experiments seeking to drive collisionless shocks, for example, one might use a 5- $\mu$ m-thick target having $\sigma=0.0012 \mathrm{~g} / \mathrm{cm}^{2}$ with $L_{o}=660 \mu \mathrm{m}$ at $t_{o}=2 \mathrm{~ns}$, and observe the system at $10 \mathrm{~ns}$ when it is still planar. Fig. 3(a) shows this case. In experiments seeking to produce spherical blast waves one might use a $26-\mu \mathrm{m}$-thick target of $5 \mathrm{~mm}$ diameter, having $M=0.0012 \mathrm{~g}$, with $L_{o}=6.6 \mathrm{~mm}$ at $t_{o}=20 \mathrm{~ns}$, and observe the spherically expanding system at $50 \mathrm{~ns}$. Fig. 3(b) shows this case. In both cases shown, the right hand edge of the figure corresponds to a velocity of about $1000 \mathrm{~km} / \mathrm{s}$.

The other relevant case is one in which heat deposition stops at some time but heat conduction remains strong enough to keep $T$ constant in space. This case has been treated in various limits by LR, QK, and HL. (One may note that all three previous papers incorrectly state that the expansion is adiabatic after the heating stops, which is not the case because there is heat conduction. QK also do not mention that their expansion is mass limited, although this is the case in their solutions.) HL, in their Eqs. (20)-(24), include solutions for the time dependence of the scale length and for the relation of temperature and scale length. These solutions require that one know the scale length $L_{o}$, the temperature $T_{o}$, and $\partial L / \partial t$ at time $t_{o}$, along with the total mass $M$.

For the aid of readers who may at some point seek to connect these papers, and to consider whether their assumptions are sufficiently general for some given application, some discussion of the relevant equation of state properties may be useful. One writes the pressure as $p=\rho R T$ and the specific internal energy as $\epsilon=C_{v} T$, where $T$ varies in time but not space, and we take the gas constant $R$ to in fact be constant (a simplification). Identifying this as an "isothermal" case, one might be tempted to take the polytropic index $\gamma$ to be 1 . If this were the case, then the results in the previous papers would be incorrect. However, in this case, there is not only one $\gamma$. It is the "acoustic $\gamma$ " whose value is one. ${ }^{4}$ The internal energy per particle remains $(n / 2) k_{B} T$, where $n$ is the number of degrees of freedom, which is 3 for constant $R$. The corresponding "shock $\gamma$ " is $\gamma_{s}=1+2 / n=5 / 3$, and the specific heat is $C_{v}=\partial(3 R T / 2)$ $/\left.\partial T\right|_{v}=3 R / 2$. These results with the above profiles enable us to reduce the equation for conservation of energy,

$$
\left.\frac{\partial}{\partial t}\left(\rho \epsilon+\frac{\rho u^{2}}{2}\right)+\nabla \cdot\left(\rho u\left(\epsilon+u^{2} / 2\right)+p u\right)\right)=0,
$$

to

$$
T L^{\prime}+(3 / 2) L T^{\prime}=0
$$

for the planar case, in agreement with Eq. (7) of LR (Ref. 6) for no heating and to

$$
T L^{\prime}+(1 / 2) L T^{\prime}=0
$$

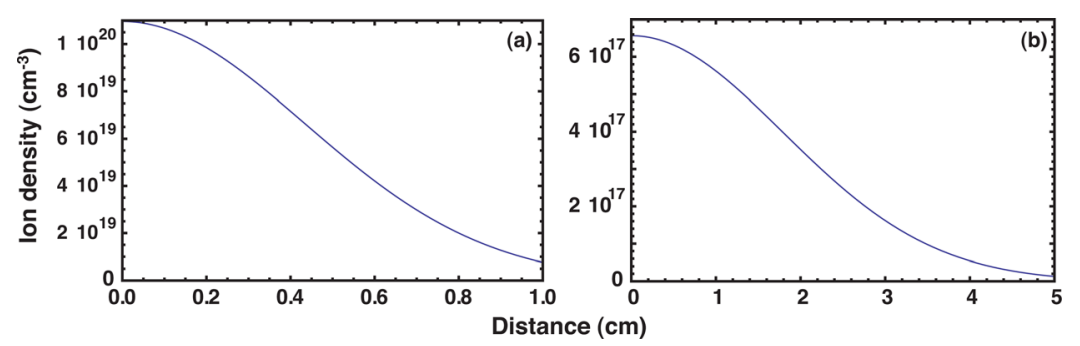

FIG. 3. (Color online) The ion density is shown for two isothermal cases corresponding to potential NIF experiments described in the text. (a) planar and (b) spherical. 
for the spherical case. These are consistent with the general result shown in HL.

This work was funded by the DOE/NNSA-DS and DOE/SC-OFES Joint Program in High-Energy-Density Laboratory Plasmas, Grant No. DE-FG52-09NA29548, by the Predictive Sciences Academic Alliances Program in DOE/NNSA-ASC via Grant No. DEFC52-08NA28616, and by the National Laser User Facility Program, Grant No. DEFG52-09NA29034, and by the University of Michigan.

${ }^{1}$ J. M. Dawson, Phys. Fluids 7, 419 (1964).

${ }^{2}$ S. A. Colgate and R. H. White, Astrophys. J. 143, 626 (1966).
${ }^{3}$ Y. B. Zeldovich and Y. P. Razier, Physics of Shock Waves and HighTemperature Hydrodynamic Phenomena, 2002 ed. (Academic, New York/Dover, 1966), Vol. 1

${ }^{4}$ R. P. Drake, High Energy Density Physics: Fundamentals, Inertial Fusion and Experimental Astrophysics (Springer-Verlag, Berlin, 2006).

${ }^{5}$ N. Qi and M. Krishnan, Phys. Fluids B 1, 1277 (1989).

${ }^{6}$ R. A. London and M. D. Rosen, Phys. Fluids 29, 3813 (1986).

${ }^{7}$ J. H. Hunter and R. A. London, Phys. Fluids 31, 3102 (1988).

${ }^{8}$ R. P. Drake, D. W. Phillion, K. Estabrook, R. E. Turner, R. L. Kauffman, and E. M. Campbell, Phys. Fluids B 1, 1089 (1989).

${ }^{9}$ L. Ferrario, J. Plasma Phys. 64, 1 (2000).

${ }^{10}$ P. Mora, Phys. Rev. Lett. 90, 185002 (2003).

${ }^{11}$ A. Beck and F. Pentellini, Plasma Phys. Controlled Fusion 51, 015004 (2009).

${ }^{12}$ W. J. Hogan, E. I. Moses, B. E. Warner, M. S. Sorem, and J. M. Soures, Nucl. Fusion 41, 567 (2000). 